\title{
ORIGINAL
}

\section{EFECTO DEL CALOR SOBRE EL NÚMERO DE URGENCIAS HOSPITALARIAS EN LA REGIÓN DE MURCIA DURANTE LOS VERANOS DEL PERÍODO 2000-2005 Y SU USO EN LA VIGILANCIA EPIDEMIOLÓGICA}

\author{
Rocío García-Pina (1), Aurelio Tobías Garcés (2), José Sanz Navarro (3), Carmen Navarro Sánchez (1) \\ y Ana García-Fulgueiras (1) \\ (1) Servicio de Epidemiología. Consejería de Sanidad de Murcia. \\ (2) Escuela Nacional de Sanidad. Instituto de Salud Carlos III. Madrid. \\ (3) Servicio de Sanidad Ambiental. Consejería de Sanidad de Murcia.
}

\section{RESUMEN}

Fundamento: el efecto de las temperaturas sobre la mortalidad ha sido estudiado más en profundidad que el efecto sobre la morbilidad. En Murcia se monitorizan el número de urgencias diarias y los casos de golpes de calor atendidos en los hospitales de la Región. Objetivo: valorar el efecto de la temperatura sobre el número de urgencias hospitalarias y la utilidad de estos indicadores para vigilar la morbilidad por calor.

Métodos: se ha estudiado el efecto de la temperatura sobre el número de urgencias en verano (periodo 2000-2005), estimando el incremento porcentual de urgencias cuando se superan los umbrales establecidos por el Ministerio de Sanidad y Consumo ( $\mathrm{T}^{\mathrm{a}}$ máxima $38^{\circ} \mathrm{C}$ y $\mathrm{T}^{\mathrm{a}}$ mínima $22,4^{\circ} \mathrm{C}$ ) y por cada grado de aumento de temperatura, expresado en Riesgo Relativo (RR) con un IC95\%. Se han cotejado los golpes de calor comunicados con los ingresos registrados en el Conjunto Mínimo Básico de Datos al Alta Hospitalaria (CMBD).

Resultados: En 2000-2005 las urgencias se incrementaron un $1,6 \%$ en los días con más de $22,4^{\circ} \mathrm{C}$ de mínima (RR 1,016 ; IC95\% $1,0076-1,0244)$, y un $0,21 \%$ por cada grado de aumento de la mínima (RR 1,0021; IC95\% 1,0000-1,0044). El 38\% de los golpes de calor ingresados no se notificaron, y el $40 \%$ de los notificados tenían exposición laboral.

Conclusiones: La temperatura mínima podría tener un mayor efecto en Murcia que la máxima. Con los actuales umbrales, el número de urgencias diarias no parece un indicador adecuado para monitorizar el efecto de la temperatura, necesitándose información sobre el diagnóstico y la edad. Los golpes de calor aportan información parcial del impacto, pero resaltan grupos de población en riesgo menos considerados.

Palabras clave: Ola de calor. Cambio climático. Urgencias hospitalarias. Golpe de calor.

Correspondencia:

Rocío García Pina

Servicio de Epidemiología

Consejería de Sanidad de la Región de Murcia

C/Ronda de Levante $n^{\circ} 11,1^{a}$ Planta. 30008 Murcia

Correo-electrónico: rocio.garcia2@carm.es
ABSTRACT

\section{Effect of Weather Temperature on Hospital Emergencies in the Region of Murcia, Spain, throughout the 2000-2005 and its Use in Epidemiological Surveillance}

Background: The effect of the weather temperature on mortality has been studied more in depth than its effect on morbidity. In Murcia, the number of daily emergencies and the cases of heat stroke for which care is provided at the hospitals in this Region have been studied. Objective: to evaluate the effect that the weather temperature has on the number of hospital emergencies and the use of these indicators for the surveillance of hot weather-related morbidity.

Methods: The effect of the weather temperature on the number of summertime emergencies (2000-2005) has been studied by estimating the percentage increase in emergencies when the weather temperature thresholds established by the Ministry of Health and Consumer Affairs (Max. $38^{\circ} \mathrm{C}$ and Min. $22.4^{\circ} \mathrm{C}$ ) are exceeded, and by each degree of temperature rise. Results have been stated as Relative Risk (RR) with a 95\% CI. A comparison has been drawn between the heat strokes notified and the hospital admissions recorded in the Minimum Basic Data Set at Hospital Discharge (MBDS).

Results: Within the 2000-2005 period, the number of emergencies rose by $1.6 \%$ on those days when the minimum temperature for the day was above $22.4^{\mathrm{a}} \mathrm{C}$ (RR: 1.016 ; $95 \% \mathrm{CI} 1.0076$ -1.0244 ) and by $0.21 \%$ for each degree of rise in the minimum temperature for the day (RR: $1.0021,95 \%$ CI 1.0000-1.0044). A total of $38 \%$ of the heat strokes admitted to hospital were not reported, of which $40 \%$ had occupational exposure.

Conclusions: The minimum temperature for the day could have a greater effect in Murcia than the maximum for the day. Based on the current thresholds, the number of emergencies/day does not seem to be a suitable indicator for monitoring the effect of the weather temperature, information on the diagnosis and the age being needed. Heat strokes provide partial information on the impact, but highlight less-considered population groups at risk.

Key words: Heat wave. Climatic change. Emergency activity. Heat stroke. 


\section{INTRODUCCIÓN}

En el cuarto Informe de Evaluación del Grupo Intergubernamental de Expertos en Cambio Climático se reconoce por primera vez que el calentamiento global es innegable, que sus consecuencias son ya muy importantes, y que "muy probablemente" este calentamiento sea debido a causas antropogénicas $^{1,2}$. Las últimas estimaciones sobre el cambio climático en Europa apuntan a que la zona meridional es una de las más vulnerables ${ }^{2-4}$. El impacto sobre la salud es evidente: sólo durante la ola de calor que azotó Europa en el verano de 2003 se produjo un exceso de mortalidad de más de 50.000 muertes $^{5}$. En España se estimó un exceso de mortalidad general del $8 \%$ que afectó exclusivamente a las personas mayores de 65 años (incremento de mortalidad del $15 \%$ ), produciéndose el mayor impacto durante el mes de agosto $^{6}$.

Para medir el impacto del calor sobre la salud se ha estudiado la relación de la temperatura y la mortalidad general. Se sabe que esta relación no es lineal sino que adopta una forma de $\mathrm{J}$ (mayor impacto de las temperaturas extremas) y que tiene un efecto retardado de 1 a 3 días después de la elevación de las temperaturas ${ }^{7}$. El grado de adaptación de la población parece jugar un papel importante, ya que el mayor exceso de mortalidad se registra a partir de un umbral de temperatura menor en las latitudes más frías (umbral de $26,2^{\circ} \mathrm{C}$ en A Coruña frente a $\operatorname{los} 41,2^{\circ} \mathrm{C}$ en Córdoba) ${ }^{8}$.

La magnitud del impacto del exceso de temperatura sobre la morbilidad no se ha estudiado con tanta profundidad, a pesar de que en países como Francia los servicios de urgencias de los hospitales presentaron un importante incremento de la presión asistencial durante la ola de calor de $2003^{9,10}$. En olas de calor previas se estimó un incremento general del
$11 \%$ en el número de ingresos ${ }^{11}$, similar al encontrado en un hospital de Barcelona durante los meses más cálidos del año 2003 comparado con $2002^{12}$. Otros estudios no han encontrado un impacto simi$\operatorname{lar}^{13,14}$. Por esta razón la relación entre ambos fenómenos requiere de estudios adicionales.

El incremento de la temperatura puede asociarse con la aparición de golpe de calor, que es un síndrome clínico grave y en ocasiones letal. Se produce a causa del fallo de los mecanismos de la termorregulación y puede afectar tanto a personas con patología de base como a personas sanas. Ésta parece ser una causa de morbimortalidad de cierta importancia cuando se registran excesos de temperaturas, aunque no la más importante $\mathrm{e}^{11,14,15}$, pero su monitorización podría ser de utilidad en la vigilancia del impacto sobre la salud de las personas. En la Región de Murcia la prevalencia de algunos de los factores de riesgo relacionados con la morbimortalidad producida por el exceso de temperaturas (diabetes mellitus, obesidad, etc) es especialmente elevada comparada con el resto del territorio estatal $^{16-18}$.

En Mayo de 2004 se instauró en todas las Comunidades Autónomas (CCAA) el Plan de Acciones Preventivas contra los Efectos del Exceso de Temperaturas sobre la Salud, elaborado por el Ministerio de Sanidad y Consumo (MSC). Según el mismo, la vigilancia del riesgo de temperaturas extremas se lleva a cabo mediante un sistema de monitorización meteorológico con diferentes niveles de alerta y respuesta. Los niveles de alerta, que determinan el riesgo de "ola de calor", se establecen en función del número de días que se espera que se vayan a superar simultáneamente los valores umbrales de temperaturas máximas y mínimas calculados para cada provincia ${ }^{19}$. Esta vigilancia se complementa con la monitorización del exceso de mortalidad asociado a elevacio- 
nes de las temperaturas, cuya metodología ha sido previamente publicada ${ }^{6,20}$. Este sistema aporta información para el conjunto de España y para niveles autonómi$\cos$ y municipales, aunque no con total cobertura, ya que se basa en la información aportada por los Registros Civiles informatizados (en Murcia se monitorizan 11 de los 45 municipios), y además presenta cierto retraso ${ }^{20}$.

En la Región de Murcia desde el año 2004 se monitorizan además el número de urgencias diarias y el número de casos atendidos por golpe de calor de todos los hospitales públicos, estudiándose su relación con el exceso de temperaturas. Se optó por analizar el número personas atendidas en los servicios de urgencias (independientemente de que ingresen o no) porque éstos podían verse especialmente afectados por situaciones de alerta de salud pública, y porque era posible obtener información diaria actualizada de todos los hospitales públicos. A diferencia de los estudios basados en ingresos hospitalarios (CMBD) y en mortalidad, este indicador presentaría a priori una mayor sensibilidad (al no restringirse a los casos de mayor gravedad), una buena cobertura, y permitiría obtener información en tiempo real.

El objetivo de este estudio es valorar la utilidad del número de urgencias diarias y de los casos de golpes de calor en la vigilancia de la morbilidad por exceso de temperaturas en la Región de Murcia durante el período 2000-2005.

\section{MATERIAL Y MÉTODOS}

Se monitorizó el número de urgencias diarias atendidas en los 9 hospitales del Servicio Murciano de Salud desde el 1 de junio hasta el 31 de septiembre para el periodo 2000-2005, sin incluir las urgencias materno-infantiles. También desde el año 2004 se monitorizaron los casos de golpe de calor atendidos en urgencias o ingresados que notifican todos los Hospitales de la Región (públicos y privados), realizándose una revisión anual de los ingresos registrados en el CMBD por este motivo.

Los valores de las temperaturas diarias máximas y mínimas registradas en verano para los años 2000 al 2005 fueron proporcionadas por la Estación Meteorológica de Guadalupe del Instituto Nacional de Meteorología (INM). Con ellas se calculó el número de días que se superaron los umbrales de temperaturas para Murcia establecidos por el $\mathrm{MSC}\left(38^{\circ} \mathrm{C}\right.$ para la máxima y $22,4^{\circ} \mathrm{C}$ para la mínima $)^{18,19}$. Este cálculo se realizó para la superación de los umbrales de la máxima y la mínima simultáneamente y para la superación de los umbrales de temperatura máxima o mínima de forma aislada. Para las estaciones de clima suave con baja oscilación térmica diaria, como nuestro caso, los valores umbrales se han establecido en base al percentil 95\% de la serie histórica de temperaturas máximas absolutas y mínimas diarias registradas en los meses de verano de años anteriores ${ }^{8}$.

Análisis estadístico: La relación entre el número de urgencias diarias atendidas y las temperaturas máximas y mínimas diarias registradas se estudió utilizando la regresión de Poisson, evaluando los posibles efectos retardados de hasta una semana. Se ajustaron los componentes de tendencia y estacionalidad utilizando términos lineales y cuadráticos para la tendencia y términos sinusoidales de hasta segundo orden para la estacionalidad ${ }^{21,22}$. Se tuvo en cuenta el efecto del día de la semana y de los días festivos mediante la introducción de variables indicadoras en los modelos. Se ajustó por el efecto del incremento de la temperatura en varios días consecutivos, de la temperatura máxima sobre la mínima, y de otras variables 
meteorológicas (humedad relativa), así como la ocurrencia de eventos epidemiológicos importantes (el brote de Legionella pneumophila ${ }^{23}$ ocurrido en la Región de Murcia en julio de 2001, y el producido por Salmonella enteritidis tipo Hadar debido al consumo de pollo precocina$\mathrm{do}^{24}$, ocurrido en España en julio de 2005). Se calculó el riesgo de demanda asistencial urgente expresado como el riesgo relativo (RR) del número de urgencias diarias atendidas en los días que se sobrepasan los umbrales de temperatura mínima y máxima frente a los que no, con un nivel de confianza del $95 \%$. Adicionalmente se calculó el RR del número de urgencias por cada incremento de un grado centígrado de temperatura, es decir, sin tener en cuenta dichos umbrales. Los RR se expresaron como el incremento porcentual del número de urgencias al superarse los umbrales en el primer caso, $\mathrm{y}$ al elevarse un grado la temperatura en el segundo. Las variables incluidas en el modelo se seleccionaron en base al nivel de significación individual, y conjuntamente a partir de las variables que minimizaban el Criterio de Información Bayesiano de Schwarz (BIC) ${ }^{25}$. Una vez seleccionado el modelo de mejor ajuste, se corrigió la posible existencia de sobredispersión con el parámetro de sobredispersión. Los análisis se realizaron utilizando el programa estadístico Stata 7.0 (StataCorp, College Station, TX).

Para evaluar la monitorización de los golpes de calor atendidos se cotejó, para el verano de cada uno de los años estudiados, el número de casos comunicados por los hospitales con los ingresos hospitalarios registrados en el CMBD, seleccionando como motivo de ingreso el código 992.0 de la CIE 9-MC (golpe de calor). Se calculó el porcentaje de casos por golpe de calor que precisaron ingreso en los días de superación de alguno de los umbrales de temperatura (datos procedentes del CMBD para el periodo 2000-2005).

\section{RESULTADOS}

En la figura 1 se muestran las medias mensuales de la demanda asistencial urgente y las temperaturas máxima y mínima diarias registradas en los veranos de los años 2000 a 2005. En la tabla 1 se muestra el número de días en los que se sobrepasaron los umbrales de temperaturas y la proporción de demanda asistencial urgente que fue atendida durante estos días sobre el total de urgencias atendidas en verano.

En los veranos de 2000 a 2002 y de 2004 a 2005 las temperaturas medias registradas fueron inferiores a las de 2003, sobre todo en los 2 primeros meses (figura 1). Los umbrales de temperaturas mínima y máxima se superaron simultáneamente con menor frecuencia en el periodo 2000-2002 que en el periodo posterior 2003-2005. Asimismo el porcentaje de urgencias atendidas durante los días en que se superaron simultáneamente los umbrales de temperatura máxima y mínima en el periodo 2000-2002 también fue menor (tabla 1).

En el verano del año 2003 se alcanzó el máximo de urgencias atendidas al día en los hospitales públicos (2.244) durante el periodo 2000-2005. Siguiendo la definición de "ola de calor" del MSC, las temperaturas máximas y mínimas diarias sobrepasaron simultáneamente sus umbrales en 5 ocasiones (tabla 1). Sin embargo, fueron las temperaturas mínimas diarias las que sobrepasaron con más frecuencia su umbral (35 veces), habiéndose atendido en aquellos días el 28,6\% del total de urgencias de todo el verano. En el mes de julio de 2003 se alcanzaron los valores más elevados de todo el verano, con $41^{\circ} \mathrm{C}$ de máxima y $25,2^{\circ} \mathrm{C}$ de mínima (figura 1 ), y la temperatura mínima sobrepasó los $22,4^{\circ} \mathrm{C}$ durante 14 días consecutivos. La media de la temperatura mínima y máxima en aquel verano fue la más alta del periodo de estudio $\left(21,0^{\circ} \mathrm{C}\right.$ y $33,7^{\circ} \mathrm{C}$, respectivamente). 


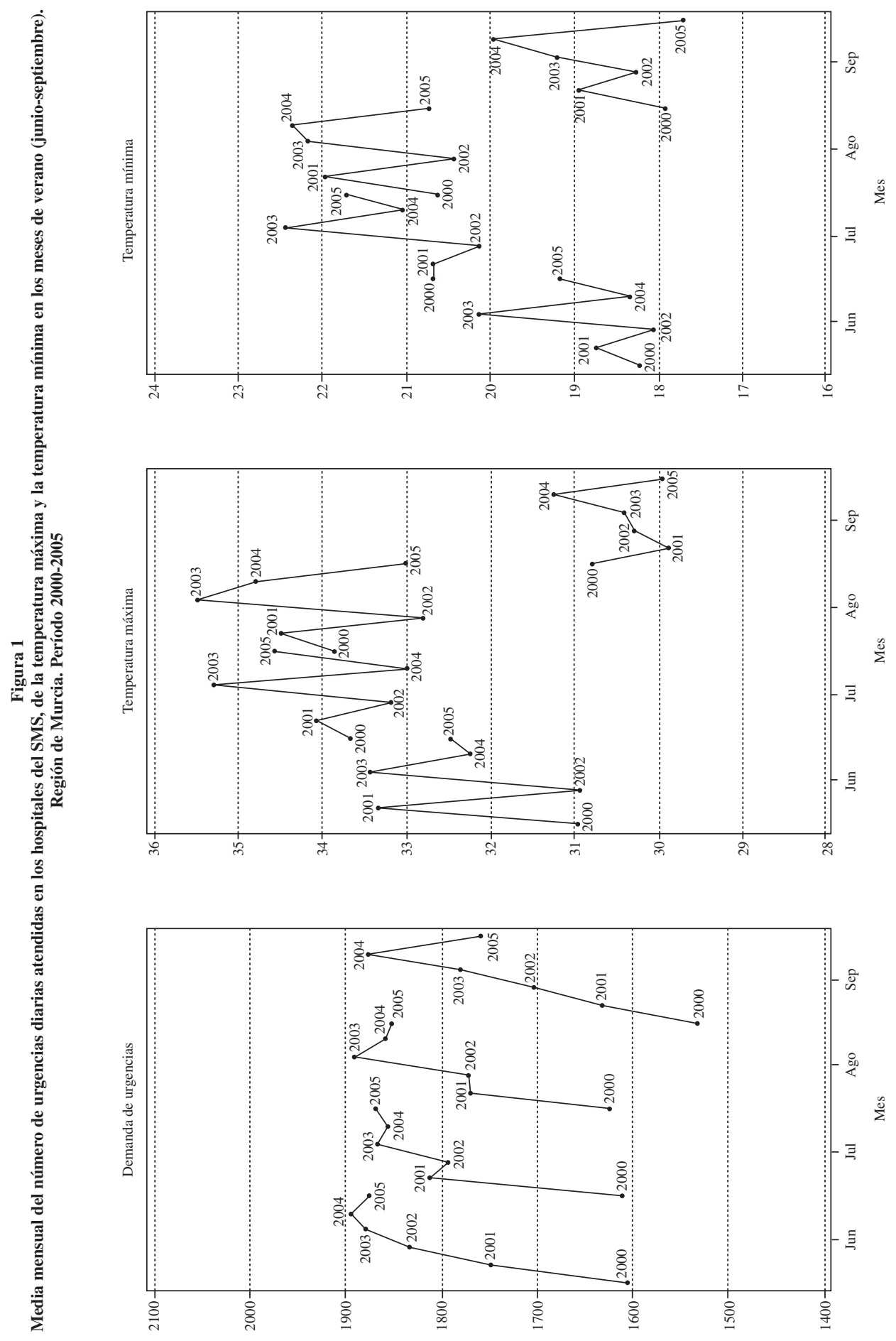

Rev Esp Salud Pública 2008, Vol. 82, N. ${ }^{\circ} 2$ 
Tabla 1

Riesgo de demanda asistencial urgente en los días en que se sobrepasan los umbrales de temperatura máxima y mínima. Región de Murcia. Periodo junio-septiembre. Años 2000-2005

\begin{tabular}{|c|c|c|c|c|c|}
\hline Año & $\begin{array}{l}\text { Número días } \\
\text { sobre umbral }\end{array}$ & $\begin{array}{l}\% \text { urgencias en } \\
\text { días sobre umbral* }\end{array}$ & $\begin{array}{c}\text { Humedad } \\
\text { relativa media }\end{array}$ & $\mathbf{R R}$ & IC95\% \\
\hline $\begin{array}{l}2000 \\
\text { Mín }=22,4^{\circ} \mathrm{C} \\
\text { Máx }=38,0^{\circ} \mathrm{C} \\
\text { Mín }=22,4^{\circ} \mathrm{C} \text { y Máx }=38,0^{\circ} \mathrm{C}\end{array}$ & $\begin{array}{l}8 \\
5 \\
1\end{array}$ & $\begin{array}{l}6,73 \\
4,07 \\
0,97\end{array}$ & $\begin{array}{l}51,8 \\
49,7 \\
55,0\end{array}$ & $\begin{array}{l}1,0065 \\
1,0086 \\
1,0005\end{array}$ & $\begin{array}{l}(0,9786-1,0353) \\
(0,9729-1,0457) \\
(0,9545-1,0486)\end{array}$ \\
\hline $\begin{array}{l}2001 \\
\text { Mín }=22,4^{\circ} \mathrm{C} \\
\text { Máx }=38,0^{\circ} \mathrm{C} \\
\text { Mín }=22,4^{\circ} \mathrm{C} \text { y Máx }=38,0^{\circ} \mathrm{C}\end{array}$ & $\begin{array}{r}18 \\
7 \\
1\end{array}$ & $\begin{array}{r}14,98 \\
5,74 \\
0,82\end{array}$ & $\begin{array}{l}59,7 \\
34,4 \\
43,2\end{array}$ & $\begin{array}{l}1,0167 \\
0,9858 \\
0,9802\end{array}$ & $\begin{array}{l}(0,9949-1,0390) \\
(0,9576-1,0148) \\
(0,9355-1,0272)\end{array}$ \\
\hline $\begin{array}{l}2002 \\
\text { Mín }=22,4^{\circ} \mathrm{C} \\
\text { Máx }=38,0^{\circ} \mathrm{C} \\
\text { Mín }=22,4^{\circ} \mathrm{C} \text { y Máx }=38,0{ }^{\circ} \mathrm{C}\end{array}$ & $\begin{array}{l}7 \\
0 \\
0\end{array}$ & $\begin{array}{l}5,87 \\
0,00 \\
0,00\end{array}$ & 64,0 & 1,0233 & $(0,9959-1,0514)$ \\
\hline $\begin{array}{l}2003 \\
\text { Mín }=22,4^{\circ} \mathrm{C} \\
\text { Máx }=38,0^{\circ} \mathrm{C} \\
\text { Mín }=22,4^{\circ} \mathrm{C} \text { y Máx }=38,0^{\circ} \mathrm{C}\end{array}$ & $\begin{array}{r}35 \\
5 \\
5\end{array}$ & $\begin{array}{r}28,56 \\
4,22 \\
4,35\end{array}$ & $\begin{array}{l}61,4 \\
49,5 \\
50,7\end{array}$ & $\begin{array}{l}1,0095 \\
1,0225 \\
0,9880\end{array}$ & $\begin{array}{l}(0,9895-1,0299) \\
(0,9834-1,0632) \\
(0,9558-1,0213)\end{array}$ \\
\hline $\begin{array}{l}2004 \\
\text { Mín }=22,4^{\circ} \mathrm{C} \\
\text { Máx }=38,0^{\circ} \mathrm{C} \\
\text { Mín }=22,4^{\circ} \mathrm{C} \text { y Máx }=38,0^{\circ} \mathrm{C}\end{array}$ & $\begin{array}{r}23 \\
3 \\
4\end{array}$ & $\begin{array}{r}18,73 \\
2,59 \\
2,58\end{array}$ & $\begin{array}{l}65,3 \\
48,2 \\
50,4\end{array}$ & $\begin{array}{l}1,0111 \\
1,0176 \\
1,0120\end{array}$ & $\begin{array}{l}(0,9930-1,0295) \\
(0,9778-1,0590) \\
(0,9826-1,0422)\end{array}$ \\
\hline $\begin{array}{l}2005 \\
\text { Mín }=22,4^{\circ} \mathrm{C} \\
\text { Máx }=38,0^{\circ} \mathrm{C} \\
\text { Mín }=22,4^{\circ} \mathrm{C} \text { y Máx }=38,0^{\circ} \mathrm{C}\end{array}$ & $\begin{array}{r}23 \\
5 \\
1\end{array}$ & $\begin{array}{r}19,04 \\
4,21 \\
0,91\end{array}$ & $\begin{array}{l}58,3 \\
50,7 \\
37,2\end{array}$ & $\begin{array}{l}1,0063 \\
0,9910 \\
0,9622\end{array}$ & $\begin{array}{l}(0,9903-1,0225) \\
(0,9597-1,0233) \\
(0,9310-0,9945)\end{array}$ \\
\hline $\begin{array}{l}\text { TOTAL } \\
\text { Mín }=22,4^{\circ} \mathrm{C} \\
\text { Máx }=38,0^{\circ} \mathrm{C} \\
\text { Mín }=22,4{ }^{\circ} \mathrm{C} \text { y Máx }=38,0^{\circ} \mathrm{C}\end{array}$ & $\begin{array}{r}114 \\
25 \\
12\end{array}$ & $\begin{array}{r}15,95 \\
3,46 \\
1,14\end{array}$ & $\begin{array}{l}60,78 \\
45,41 \\
49,21\end{array}$ & $\begin{array}{l}1,0160 \\
1,0045 \\
0,9910\end{array}$ & $\begin{array}{l}(1,0076-1,0244) \\
(0,9887-1,0206) \\
(0,9755-1,0068)\end{array}$ \\
\hline
\end{tabular}

* \% de urgencias atendidas en días que se superó el umbral del MSC sobre el total de urgencias atendidas en todo el verano.

Por otro lado, en la figura 1 se observa que la demanda asistencial urgente durante los meses de verano presentó una tendencia ascendente a lo largo del periodo 20002005, con un incremento total del $15 \%$. Paralelamente la población residente en Murcia se incrementó un 16,2\% en el periodo 2000-2005, según la información disponible en el Padrón Municipal de habitantes. Dicho incremento parece ser independiente del grado de envejecimiento de la población, ya que su distribución por grupos de edad no se modificó prácticamente en los 5 años. El ascenso medio anual fue del 2,6\% para las urgencias hospitalarias, siendo esta tendencia más marcada en el verano de 2001 (incremento del 9,3\% respecto al año 2000), y mantenida hasta 2003. En los veranos de 2004 y 2005 esta tendencia se estabilizó. También hay que tener en cuenta que la demanda asistencial urgente suele incrementarse a partir del mes de junio y alcan- zar su pico máximo en julio o agosto para decaer en septiembre (figura 1).

La figura 2 muestra la relación de las series de temperatura y urgencias ajustadas por sus componentes de tendencia y estacionalidad, siendo la pendiente algo más pronunciada para la mínima en la mayoría de los años, con excepción del año 2003 donde es la máxima la que presenta una mayor pendiente.

En la tabla 1 se muestra el riesgo de la demanda asistencial urgente producido en los días en los que se sobrepasaron los umbrales de temperaturas con respecto a los días en que no se sobrepasaron. El modelo que mejor se ajusta a los datos registrados es aquel que contempla el efecto retardado a los 6 días de sobrepasarse los umbrales, ya que el efecto del incremento de la temperatura mínima en la región de Murcia parece ser mayor que el de la máxi- 


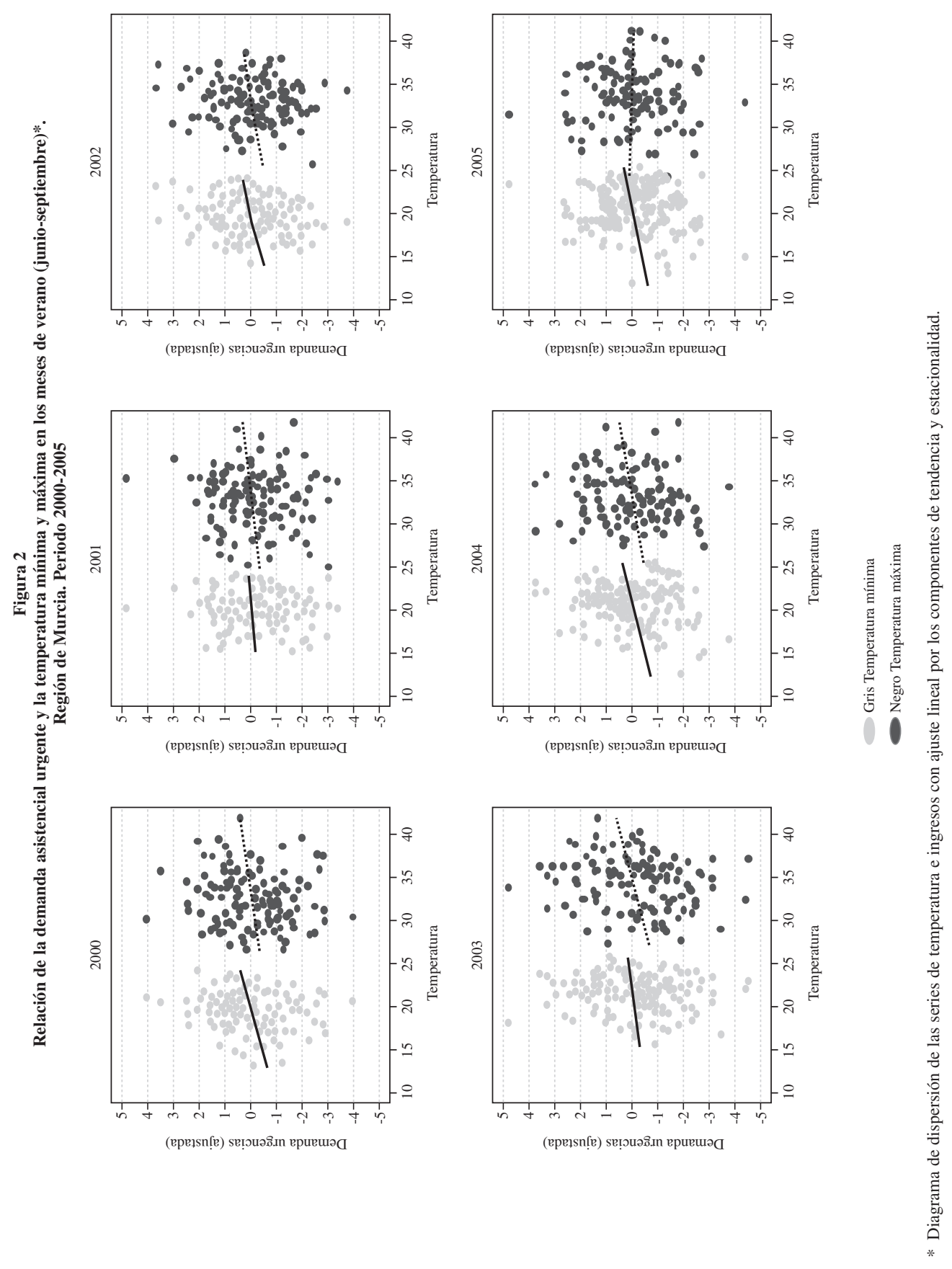


ma, y podría tener un efecto sobre la salud más retardado. Por tanto, los riesgos que figuran en la tabla hacen referencia a este efecto. Durante los veranos de 2000 a 2005 se produjo un pequeño incremento de la demanda asistencial urgente (del 1,6\%) asociado a los días en que se sobrepasó el umbral de temperatura mínima de $22,4{ }^{\circ} \mathrm{C}$ (RR 1,016; IC 95\% 1,008-1,024). En el año 2003 se registró el mayor efecto de las altas temperaturas sobre las urgencias hospitalarias, con un incremento del $2,2 \%$ en el número de urgencias diarias cuando se sobrepasó el umbral de temperatura máxima de $38^{\circ} \mathrm{C}$, aunque no llegó a alcanzar la significación estadística (RR 1,022; IC95\% 0,983-1,063).

Al eliminar del análisis el año 2003, por ser un año anormalmente caluroso, la magnitud del efecto encontrada en relación a la temperatura mínima para todo el periodo tuvo los siguientes valores cuando se sobre pasaron los $22,4^{\circ} \mathrm{C}$ de mínima: RR 1,0156 IC95\% 1,0060-1,0253. El incremento de urgencias en los días en que se sobrepasaron $\operatorname{los} 38^{\circ} \mathrm{C}$ de máxima no resultó significativo.

En la tabla 2 se presenta el riesgo de la demanda asistencial urgente producido por

Tabla 2

Riesgo de demanda asistencial urgente por cada grado de incremento de temperatura. Región de Murcia. Período junio-septiembre. 2000-2005

\begin{tabular}{|c|c|c|c|c|c|}
\hline \multirow{2}{*}{ Años } & \multirow{2}{*}{ Temperatura } & \multicolumn{2}{|c|}{ Modelo para temperatura mínima } & \multicolumn{2}{|c|}{ Modelo para temperatura máxima } \\
\hline & & $\mathbf{R R}$ & IC $95 \%$ & $\mathbf{R R}$ & IC $95 \%$ \\
\hline 2000 & $\begin{array}{l}\text { Mínima (t) } \\
\text { Mínima (t-1) } \\
\text { Mínima (t-6) } \\
\text { Máxima (t) } \\
\text { Máxima (t-1) } \\
\text { Máxima (t-6) }\end{array}$ & $\begin{array}{l}1,0049 \\
1,0038 \\
1,0003\end{array}$ & $\begin{array}{l}(1,0007-1,0091) \\
(0,9994-1,0083) \\
(0,9959-1,0048)\end{array}$ & $\begin{array}{l}1,0021 \\
1,0002 \\
1,0005\end{array}$ & $\begin{array}{l}(0,9996-1,0047) \\
(0,9976-1,0027) \\
(0.9979-1,0031)\end{array}$ \\
\hline 2001 & $\begin{array}{l}\text { Mínima (t) } \\
\text { Mínima (t-1) } \\
\text { Mínima (t-6) } \\
\text { Máxima (t) } \\
\text { Máxima (t-1) } \\
\text { Máxima (t-6) }\end{array}$ & $\begin{array}{l}0,9999 \\
0,9982 \\
1,0031\end{array}$ & $\begin{array}{l}(0,9954-1,0045) \\
(0,9937-1,0029) \\
(0,9983-1,0079)\end{array}$ & $\begin{array}{l}1,0011 \\
1,0009 \\
0,9991\end{array}$ & $\begin{array}{c}(0,9986-1,0036) \\
(0,9982-1,0035) \\
(0,9964-1,0018)\end{array}$ \\
\hline 2002 & $\begin{array}{l}\text { Mínima (t) } \\
\text { Mínima (t-1) } \\
\text { Mínima (t-6) } \\
\text { Máxima (t) } \\
\text { Máxima (t-1) } \\
\text { Máxima (t-6) }\end{array}$ & $\begin{array}{l}1,0046 \\
1,0029 \\
1,0032\end{array}$ & $\begin{array}{l}(1,0005-1,0087) \\
(0,9987-1,0072) \\
(0,9989-1,0075)\end{array}$ & $\begin{array}{l}1,0024 \\
1,0048 \\
1,0032\end{array}$ & $\begin{array}{c}(0,9991-1,0057) \\
(1,0016-1,0081) \\
(0,9989-1,0075)\end{array}$ \\
\hline 2003 & $\begin{array}{l}\text { Mínima (t) } \\
\text { Mínima (t-1) } \\
\text { Mínima (t-6) } \\
\text { Máxima (t) } \\
\text { Máxima (t-1) } \\
\text { Máxima (t-6) }\end{array}$ & $\begin{array}{l}1,0026 \\
1,0019 \\
1,0040\end{array}$ & $\begin{array}{l}(0,9968-1,0084) \\
(0,9956-1,0081) \\
(0,9978-1,0102)\end{array}$ & $\begin{array}{l}1,0050 \\
1,0070 \\
1,0006\end{array}$ & $\begin{array}{l}(1,0009-1,0092) \\
(1,0028-1,0112) \\
(0,9962-1,0051)\end{array}$ \\
\hline 2004 & $\begin{array}{l}\text { Mínima (t) } \\
\text { Mínima (t-1) } \\
\text { Mínima (t-6) } \\
\text { Máxima (t) } \\
\text { Máxima (t-1) } \\
\text { Máxima (t-6) }\end{array}$ & $\begin{array}{l}1,0051 \\
1,0057 \\
1,0040\end{array}$ & $\begin{array}{l}(1,0013-1,0090) \\
(1,0019-1,0095) \\
(1,0000-1,0080)\end{array}$ & $\begin{array}{l}1,0019 \\
1,0035 \\
1,0016\end{array}$ & $\begin{array}{c}(0,9995-1,0044) \\
(1,0011-1,0060)^{*} \\
(0,9991-1,0041)\end{array}$ \\
\hline 2005 & $\begin{array}{l}\text { Mínima (t) } \\
\text { Mínima (t-1) } \\
\text { Mínima (t-6) } \\
\text { Máxima (t) } \\
\text { Máxima (t-1) } \\
\text { Máxima (t-6) }\end{array}$ & $\begin{array}{l}1,0031 \\
1,0011 \\
1,0035\end{array}$ & $\begin{array}{l}(0,9998-1,0065) \\
(0,9976-1,0046) \\
(0,9993-1,0078)\end{array}$ & $\begin{array}{l}0,9994 \\
1,0013 \\
0,9991\end{array}$ & $\begin{array}{l}(0,9967-1,0020) \\
(0,9985-1,0039) \\
(0,9964-1,0019)\end{array}$ \\
\hline Total & $\begin{array}{l}\text { Mínima (t) } \\
\text { Mínima (t-1) } \\
\text { Mínima (t-6) } \\
\text { Máxima (t) } \\
\text { Máxima (t-1) } \\
\text { Máxima (t-6) }\end{array}$ & $\begin{array}{l}1,0042 \\
1,0032 \\
1,0040\end{array}$ & $\begin{array}{l}(1,0026-1,0058) \\
(1,0015-1,0048) \\
(1,0024-1,0057)\end{array}$ & $\begin{array}{l}1,0025 \\
1,0031 \\
1,0016\end{array}$ & $\begin{array}{l}(1,0014-1,0036) \\
(1,0019-1,0042) \\
(1,0004-1,0027)\end{array}$ \\
\hline
\end{tabular}


cada grado centígrado de aumento en las temperaturas mínima y máxima del día estudiado y sus efectos retardados. Se presentan los modelos que muestran los efectos del mismo día y con un retardo de uno y 6 días para la temperatura máxima y mínima por ser los que mejor se ajustan a los datos registrados. Según los datos disponibles, en Murcia para el periodo 2000-2005 por cada grado centígrado que aumentó la temperatura máxima diaria se produjo un incremento en el número de urgencias del 0,25\% (RR 1,0025; IC95\% 1,0014-1,0036), mientras que el efecto fue ligeramente mayor por cada grado centígrado que aumentó la temperatura máxima del día anterior, produciéndose un efecto retardado del 0,31\% (RR 1,0031; IC95\% 1,00191,0042). Estos efectos son los estimados en modelos independientes, pero al tener en cuenta en un único modelo la influencia de la temperatura máxima y de la mínima de los días previos prácticamente deja de observarse el mayor efecto retardado de la máxima (tabla 3).

En cuanto a la temperatura mínima, el número de urgencias diarias se incrementó en un $0,42 \%$ por cada grado centígrado

Tabla 3

Riesgo de demanda asistencial urgente por cada grado de incremento de temperatura. Región de Murcia.

Meses junio-septiembre. Total del periodo 2000-2005

\begin{tabular}{|l|c|c|}
\hline \multirow{2}{*}{ Temperaturas } & \multicolumn{2}{|c|}{$\begin{array}{c}\text { Modelo para temperatura } \\
\text { mínima y máxima* }\end{array}$} \\
\cline { 2 - 3 } & RR & \multicolumn{1}{c|}{ IC 95\% } \\
\hline Temperatura Mínima & & \\
Mínima (t) & 1,0022 & $(1,0001,1,0044)$ \\
Mínima (t-1) & 0,9996 & $(0,9971,1,0021)$ \\
Mínima (t-2) & 0,9996 & $(0,9971,1,0021)$ \\
Mínima (t-3) & 1,0006 & $(0,9981,1,0030)$ \\
Mínima (t-4) & 1,0019 & $(0,9994,1,0043)$ \\
Mínima (t-5) & 1,0008 & $(0,9983,1,0032)$ \\
Mínima (t-6) & 1,0021 & $(1,0000,1,0041)$ \\
\hline Temperatura Máxima & & \\
Máxima (t) & 1,0013 & $(1,0000,1,0026)$ \\
Máxima (t-1) & 1,0015 & $(0,9999,1,0030)$ \\
Máxima (t-2) & 1,0001 & $(0,9983,1,0019)$ \\
Máxima (t-3) & 1,0009 & $(0,9993,1,0025)$ \\
Máxima (t-4) & 1,0005 & $(0,9989,1,0020)$ \\
Máxima (t-5) & 1,0000 & $(0,9985,1,0016)$ \\
Máxima (t-6) & 0,9998 & $(0,9983,1,0012)$ \\
\hline
\end{tabular}

* Modelo ajustado por el efecto de la temperatura de los días previos y en efecto de la máxima sobre la mínima. de temperatura mínima del día actual (RR 1,0042; IC95\% 1,0026-1,0058), y tuvo un efecto retardado de 6 días que supuso un aumento del $0,40 \%$ por cada grado centígrado de elevación de la temperatura mínima (RR 1,0040; IC95\% 1,00241,0057). Dicho efecto se mantiene al tener en cuenta la influencia de la temperatura de los días previos y de la máxima de ese mismo día sobre las urgencias (tabla 3) con un $\mathrm{RR}=1,0021$; IC $95 \%$ (1,0000-1,0041). Teniendo en cuenta el ajuste mencionado se sigue observando que el aumento de la temperatura mínima produce un mayor impacto sobre el número de urgencias que el de la máxima, aunque comparado con el modelo no ajustado disminuye su magnitud. Para todo el periodo los efectos de las temperaturas sobre la demanda asistencial urgente encontrados son pequeños, siendo los de mayor magnitud los encontrados en los años 2003 y 2004: en 2003 el mayor incremento corresponde a la máxima con un efecto retardado de un día, incrementándose el número de urgencias diarias un $0,7 \%$ por cada elevación de un grado centígrado de temperatura máxima del día anterior (RR 1,0070; IC95\% 1,0028-1,0112), y en 2004 el mayor efecto corresponde a la mínima, con un incremento del $0,51 \%$ por cada grado de elevación de la temperatura mínima (RR 1,0051; IC 95\% 1,0013-1,0090).

En cuanto a los casos de golpe de calor, según la revisión realizada a través del CMBD, en los veranos de 2000 a 2005 ingresaron en los hospitales murcianos 58 personas por esta causa (21 casos en los últimos 2 años), 9 de los cuales figuran como fallecidos al alta (4 en 2003), con una letalidad del $15,5 \%$. A lo largo de todo el periodo, los ingresos por golpe de calor coincidieron con días donde se elevaron las temperaturas máximas y/o mínimas en el 60,4\% de los casos, registrándose el mayor número de ingresos en el año 2003 (24 casos). Hubo un predominio 
masculino entre los ingresos por golpe de calor $(81 \%)$, y la media de edad de todos los casos fue de 48,6 años. Las causas de exposición a altas temperaturas se monitorizaron desde el año 2004 mediante las notificaciones de los casos de golpes de calor atendidos en los hospitales durante el verano, independientemente de que hayan precisado ingreso o no. Los hospitales han notificado un total de 20 casos de golpe de calor en los 2 años. De éstos, 13 precisaron ingreso hospitalario, lo que implicó un 38,1\% de subnotificación respecto a lo registrado en el CMBD. Del total de personas que padecieron golpe de calor a lo largo de los 2 años el $40 \%$ tenían antecedente de exposición laboral a altas temperaturas, siendo esta la categoría de riesgo más frecuente.

\section{DISCUSIÓN}

Durante el periodo de monitorización (veranos de 2000 al 2005) se ha observado que en la Región de Murcia las temperaturas mínimas superaron los umbrales de extremos térmicos con más frecuencia que las máximas, y que el número de días en los que se produjo este hecho fue cada vez mayor. Hemos encontrado un efecto del $1,6 \%$, del exceso de temperaturas sobre la demanda asistencial urgente, correspondiendo el mayor riesgo a los aumentos de la temperatura mínima, con un efecto retardado de 6 días. La magnitud del efecto encontrada concuerda con la descrita en otros países europeos, como Inglaterra, donde a priori el grado de preparación de la población para afrontar olas de calor es menor $^{13,14}$.

En general en la península Ibérica la temperatura máxima diaria parece ser la variable más relevante, jugando la temperatura mínima un papel secundario ${ }^{26,27}$. En cambio, en la Región de Murcia la temperatura mínima diaria podría tener una mayor importancia. En ella no es infre- cuente que se registren periodos de 4 o más días consecutivos sobrepasando los umbrales de la mínima, o periodos en los que, sin sobrepasar el umbral, las temperaturas se mantienen al límite durante varios días consecutivos.

En cuanto al efecto retardado encontrado, en este tipo de estudios es difícil diferenciar si los efectos encontrados en el día actual o con un retardo de un día están producidos por la propia elevación de la temperatura o por las características del sistema de información. Esto es debido a que no se recoge el número de urgencias según el momento en que la temperatura diaria alcanza su máximo o en que la persona inicia los síntomas, sino según el momento en que acude a un servicio de urgencias hospitalario, pudiendo retrasarse este hecho hasta el día siguiente. Sin embargo, esta dificultad generalmente se plantea para los retardos de un día, no influyendo sobre el efecto retardado de la mínima que hemos encontrado a los 6 días. Varios estudios han detectado incrementos de la mortalidad a los 0-3 días de la elevación de la temperatura máxima ${ }^{27,28}$, pero otros también apuntan que el efecto sobre los ingresos hospitalarios podría manifestarse más a largo plazo $^{28,29}$.

La magnitud del efecto encontrada es pequeña, lo cual es consistente con otros estudios, que apuntan a un menor impacto de las temperaturas sobre el número de ingresos urgentes que sobre la mortalidad $^{13,14,28}$. Además, la Región de Murcia fue una de las CCAA menos afectadas durante la ola de calor de 2003, en lo que a mortalidad por exceso de temperaturas se refiere ${ }^{8,26}$, tratándose de una CCAA que suele presentar altas temperaturas en verano comparada con otras ${ }^{8,22}$. Los mecanismos de adaptación individuales y el grado de preparación de la población para soportar las temperaturas extremas podrían haber jugado a favor de un menor efecto ${ }^{6,7,30}$. 
Adicionalmente hay que tener en cuenta que, bajo la definición de umbrales de temperaturas establecida por el MSC para la Región de Murcia $^{19}$ (mínima $\geq 22,4^{\circ} \mathrm{C}$ y máxima $\geq 38,0^{\circ} \mathrm{C}$ ), la elevación del índice de extremos térmicos se ha producido con poca frecuencia. Estos umbrales han sido calculados para cada provincia en base a la serie histórica de datos meteorológicos exclusivamente, y presenta ciertas limitaciones $^{31}$. En cambio, el umbral a partir del cual se producen excesos de mortalidad en la Región de Murcia es de $37,5^{\circ} \mathrm{C}^{8}$, medio grado menor que el marcado por el MSC.

Desde Junio de 2005 el Plan Nacional de Acciones Preventivas contra los Efectos del Exceso de Temperaturas sobre la Salud contempla también la monitorización de datos hospitalarios, no habiéndose establecido los umbrales de temperatura a partir de los cuales se producen incrementos del número de urgencias para cada CCAA. Un estudio en un hospital de Madrid sugiere que los umbrales calculados en base a estudios de impacto sobre la mortalidad son similares a los calculados para ingresos hospitalarios $^{28}$.

En conclusión, en la Región de Murcia y con los actuales umbrales de temperatura, nuestro estudio evidencia que el número total de urgencias diarias por sí mismo no parece ser un indicador muy adecuado para monitorizar el efecto de las temperaturas sobre la salud. Otros autores han encontrado efectos, tanto en los ingresos hospitalarios ${ }^{11,12,13,15,28,29}$ como en la mortalidad $^{6,7,32}$, sobre todo asociados a grupos de edad concretos (mayores de 6075 años de edad) y a determinadas patologías (cardiovasculares y respiratorias). Los resultados, a veces no son directamente comparables debido a la metodología utilizada, pero los patrones podrían diferir en función de la variable considerada: en el caso de la mortalidad parece asociarse más a causas cardiovasculares y en el de los ingresos hospitalarios a las enfermedades respiratorias. Existen varias teorías que podrían explicar esta diferencia de patrones, aunque son escasos los estudios comparativos al respecto ${ }^{14,28,32}$. De cualquier manera, lo que sí parece claro es que para que la vigilancia de los efectos de las temperaturas extremas sobre el número de urgencias sea útil es necesario contar con información específica sobre la edad y el diagnóstico de las personas que demanadan la atención en urgencias, actualmente no disponible en nuestra CCAA. Esta evaluación puede resultar de utilidad para otras CCAA que ya monitorizan el número de urgencias atendidas en hospitales ${ }^{33,34,35}$.

La monitorización de los golpes de calor aporta información parcial del impacto de las temperaturas sobre la salud, pero puede poner de relieve la existencia de grupos de población en riesgo habitualmente menos considerados, como los trabajadores expuestos a altas temperaturas. Se ha observado que ésta no es la principal causa de morbimortalidad en la población cuando ocurre el fenómeno llamado "ola de calor", sino que el calor afecta de forma más acusada a las personas con peor capacidad basal de adaptación a las altas temperaturas, agravando patologías subyacentes. En los países más desarrollados los grupos de población considerados con mayor riesgo son las personas mayores de 75 años, o con diversas patologías de base y es sobre ellas que se han realizado los mayores esfuerzos de prevención $^{6,11,15}$. Se debe intensificar también la prevención en otros colectivos cuya estado de salud a priori no supone una mayor vulnerabilidad.

Diferentes análisis de las tendencias meteorológicas a largo plazo subrayan que el calentamiento global es una realidad, y que influirá directamente en la salud de las poblaciones ${ }^{1,2,36,37}$. Su impacto variará en función de múltiples factores, que abarcan desde la vulnerabilidad 
física hasta la vulnerabilidad social. La monitorización de la mortalidad ha sido hasta ahora el objetivo principal de la vigilancia del impacto del exceso de temperaturas sobre la salud. Otras fuentes de información (hospitales, centros de atención primaria) nos pueden ofrecer aproximaciones diferentes a esta realidad, poniendo de relieve patrones distintos a los esperados y grupos de riesgo menos considerados. La utilidad de dichas fuentes para la vigilancia debe ser valorada en función de la información disponible. La monitorización del número total de urgencias hospitalarias, sin disponer de información más desagregada y contando con los actuales umbrales, no parece ser un indicador muy útil para medir el impacto de las temperaturas extremas en nuestra población.

\section{AGRADECIMIENTOS}

A Visitación García-Ortúzar, por su colaboración en la monitorización de los casos de golpe de calor. A Rosa López Casares por su colaboración en la vigilancia ambiental. A Lauro Hernando Arizaleta por facilitar la información procedente del CMBD. A Diego Salmerón por sus valiosos comentarios. A todas las Gerencias y Direcciones médicas de los hospitales del SMS y del 061 por la comunicación de los casos de golpe de calor y por facilitar la información sobre las urgencias atendidas.

\section{BIBLIOGRAFÍA}

1. Abanades García JC, Cuadrat Prats JM, De Castro Muñoz de Lucas M, Fernández García F, Gallastegui Zulaica C, Garrote de Marcos L, et al. El Cambio Climático en España. Estado de situación. Madrid: Ministerio de Medio Ambiente; 2007 [citado 17 de noviembre 2007]. Disponible en: www.mma.es/portal/secciones/cambio_climático

2. Grupo Intergubernamental de Expertos sobre Cambio Climático. Cambio Climático 2007.
Resumen para Responsables de Políticas. IV Informe de Evaluación. Ministerio de Medio Ambiente. Madrid, 2007 [citado 17 de noviembre 2007]. Disponible en: www.mma.es/portal/secciones/cambio_climático

3. Velázquez de Castro F. Cambio Climático y Protocolo de Kioto. Ciencia y Estrategias. Compromisos para España. Rev Esp Salud Pública. 2005; 79(2): 191-201.

4. Meehl GA, Tebaldi C. More intense, more frequent and longer lasting heat waves in the $21 \mathrm{st}$ Century. Science. 2004; 305(5686): 994-7.

5. Kotatsky T. The 2003 European heat waves. Eurosurveillance monthly release 2005 ; 10(7-8): 148171.[citado 24 marzo 2006]. Disponible en: www.eurosurveillance.org

6. Martínez Navarro F, Simón-Soria F, López-Abente G. Valoración del impacto de la ola de calor del verano de 2003 sobre la mortalidad. Gac Sanit. 2004; 18(Supl 1): 250-8.

7. Kunst AE, Looman CWN, Mackenbach JP. Outdoor air temperature and mortality in the Netherlands: a time-series analysis. Am J Epidemiol. 1993; 137: 331-41.

8. Díaz-Jiménez J, Linares-Gil C, García-Herrera R. Impacto de las temperaturas extremas sobre la salud pública: futuras acciones. Rev Esp Salud Pública. 2005; 79(2): 145-157.

9. Impact sanitaire de la vague de chaleur d'août 2003 en France. Bilan et perspectives. Département maladies chroniques et traumatismes. Département santé environement. Institut de Veille Sanitarie (INVS). Sain Maurice Cedex; 2003.

10. Claesscens YE, Taupin P, Kierzek G, Pourrita JL, Baud M, Ginsburg C, et al. How emergency departments might alert for pre-hospital heat-related excess mortality? Critical Care 2006; 10(6). [citado 05 junio 2007]. Disponible en: http://ccforum.com/content/10/6/R156

11. Semanza JC, McCullough JE, Flanders DW, Mc Geehib MA, Lumpkin JR. Excess of Hospital admissions during the July 1995 Heat Wave in Chicago. Am J Prev Med 1999; 16(4): 269-77.

12. Trejo O, Miró O, De la Red G, Collvinent B, Bragulat E, Asenjo MA, et al. Impacto de la ola de calor de verano de 2003 en un servicio de urgencias hospitalario. Med Clin (Barc). 2005; 125(6): 205-9. 
13. Johnson H, Kovats R, Mc Gregor G, Stedman J, Gibbs M, Walton H, et al. The impact of the 2003 heat wave on mortality and hospital admissions in England. Health Stat Q. 2005; 25: 6-11.

14. Kovats RS, Hajat S, Wilkinson P. Contrasting patterns of mortality and hospital admissions during hot weather and heat waves in Greater London, UK. Occup Environ Med. 2004: 61; 893-8.

15. Villamil Cajoto I, Díaz Peromingo JA, Villacian Vicedo G, Sánchez Leira J, García Suárez F, Saborido Forjan J, et al. Impacto de la ola de calor de 2003 en el Hospital de Riviera (A Coruña). Ann Med Int. 2005; 22(1): 15-20.

16. Cerezo J, Tormo MJ, Navarro C, RodríguezBarranco M, Marco R, Egea JM et al. Diabetes y obesidad en la población adulta de la Región de Murcia. Murcia: Consejería de Sanidad. Dirección General de Salud Pública. Serie Informes nº 40, 2005.

17. Tormo Díaz MJ, García Rodríguez J, Cirera Suárez Ll, Contreras Gil J, Martínez López C, Rodríguez Barranco M, et al. Epidemiología del infarto agudo de miocardio en la Región de Murcia: Estudio IBÉRICA. Murcia: Consejería de Sanidad, Dirección General de Salud Pública. Serie de informes $n^{\circ} 34,2003$.

18. Plan Nacional de Adaptación al Cambio Climático. Oficina Española de Cambio Climático S. G. para la Prevención de la Contaminación y del Cambio Climático. Ministerio de Medio Ambiente. Madrid; 2006.

19. Plan de Acciones Preventivas contra los Efectos del Exceso de Temperaturas sobre la Salud. Ministerio de Sanidad y Consumo. Madrid; 2005.

20. Simón F, Flores V, Martín I. Evolución de la mortalidad en España durante el mes de julio de 2006. Resultados del sistema de vigilancia de la mortalidad diaria. Centro Nacional de Epidemiología. Bol Epidemiol Sem. 2006;14 (7): 73-84.

21. Sáez M, Pérez-Hoyos S, Tobías A, Taurina C, Barceló MA, Ballester F. Métodos de series temporales en los estudios epidemiológicos sobre contaminación atmosférica. Rev Esp Salud Pública. 1999; 73 (2): 133-143.

22. Bowle C, Prothero D. Finding causes of seasonal diseases using time-series analysis. Int J Epidemiol. 1981; 10: 87-92.

23. García Fulgueiras AM, Navarro C, Fenoll D, García J, González-Diego P, Jiménez-Buñuales T, et al. Legionnaires' Disease Outbreak in Murcia, Spain. Emerg Infect Dis. 2003; 9(8): 915-921.

24. Legnet A. Over 2000 cases so far in Salmonella hadar outbreak in Spain associated with consumption of pre-cooked chicken. Euro Surveill. 2005; 10(7): 196-197.

25. Schwarz, G. "Estimating the dimension of a model". Annals of Statistics 1978; 6(2): 461-464.

26. Díaz J, García-Herrera R, Trigo RM, Linares C, Valente MA, De Miguel JM, et al. The Impact of the summer 2003 heat wave in Iberia: how should we measure it? Int J Biometeorol. 2006; 50: 144-53.

27. García-Herrera R, Díaz J, Triho RM, Hernández E. Extreme summer temperatures in Iberia: health impacts and associated synoptic conditions. Ann Geophys 2005; 23: 239-251.

28. Linares C, Díaz J. Impact of high temperatures on hospital admissions: comparative analysis with previous studies about mortality (Madrid). Eur J Public Health. Advanced access published November 28, 2007.

29. Lage B, Díaz J, Gestal JJ, Pajares MS, Alberdi JC. Influencia de los factores ambientales en el número de ingresos por urgencia en el complejo hospitalario "Juan Canalejo" de A Coruña: elaboración de un modelo de predicción. Rev Esp Salud Pública. $1999 ; 73$ : 45-60.

30. Hajat S, Amstrong B, Wilkinson P, Carson C. Declining vulnerability to temperature-related mortality in London over the 20th century. Am J Epidemiol. 2006; 164(1): 77-84.

31. Mirón Pérez IJ, Montero Rubio JC, Criado-Álvarez JJ, Gutiérrez Ávila G, Paredes Beato D, Mayoral Arenas $\mathrm{S}$ et al. Tratamiento y estudio de series de temperatura para su aplicación en salud pública. El caso de Castilla-La Mancha. Rev Esp Salud Pública. 2006; 80: 113-24.

32. Mastrangelo G, Hajat S, Fadda E. Contrasting patterns of hospital admissions and mortality during heat waves: are death from circulatory disease a real excess or an artefact? Med Hypotheses. 2006; 66: $1025-8$.

33. Pla d'actuació per prevenir els efectes d'una onada de calor sobre la salud. Informe de les actuacions realitzades i resultats obtinguts. Estiu 2006. Generalitat de Catalunya. Departament de Salut. Direcció General de Salut Pública. Barcelona; 2006 [citado 6 septiembre 2007]. Disponible en: http://www.gencat.net/salut/depsan/units/sanitat/pdf/pla2006calor.pdf. 
34. Plan de alerta, prevención y control de los efectos de la ola de calor sobre la salud en la Comunidad Autónoma de La Rioja. Gobierno de la Rioja. Bol Epidemiol. 2004; 197: 1423-1427. [citado 6 septiembre 2007]. Disponible en: www.larioja.org/web/centrales/salud/ber/ber_jul io_2004_n197.pdf.

35. Impacto de la temperatura durante el verano de 2005 en la actividad de urgencias y en la mortalidad en Navarra. Instituto de Salud Pública de Navarra. Boletín Informativo 2006;38:6-7. [cita- do 6 julio 2007]. Disponible en: www.cfnavarra.es/ISP/documentacion/BOL38-06.pdf.

36. Patz, J, Campbell D, Holloway T, Foley J. Impact of Regional climate change on human health. Nature. 2005; 438: 310-6.

37. Díaz J, Ballester F, López Vélez R. Impactos del cambio climático en España. Impactos sobre la salud humana. [citado agosto 2006]. Disponible en: www.mma.es/oecc/pdf/16_salud_humana_2.pdf 